\title{
Temperature Distribution in Porous Fins, Subjected to Convection and Radiation, Obtained from the Minimization of a Convex Functional
}

\author{
Maria Laura Martins-Costa $\left(\mathbb{D},{ }^{1}\right.$ Vinícius Vendas Sarmento, ${ }^{1}$ Allan Moraes de Lira, ${ }^{2}$ \\ and Rogério M. Saldanha da Gama ${ }^{2}$ \\ ${ }^{1}$ Laboratory of Theoretical and Applied Mechanics (LMTA) Mechanical Engineering Graduate Program (TEM-PGMEC), \\ Universidade Federal Fluminense, Rua Passo da Pátria, 156, Niterói, RJ 24210-240, Brazil \\ ${ }^{2}$ Mechanical Engineering Graduate Program (FEN), Universidade do Estado do Rio de Janeiro, Rua São Francisco Xavier, 524, \\ Rio de Janeiro 20550-013, Brazil
}

Correspondence should be addressed to Maria Laura Martins-Costa; laura@mec.uff.br

Received 18 June 2020; Revised 28 July 2020; Accepted 1 August 2020; Published 26 August 2020

Academic Editor: Samuele De Bartolo

Copyright (c) 2020 Maria Laura Martins-Costa et al. This is an open access article distributed under the Creative Commons Attribution License, which permits unrestricted use, distribution, and reproduction in any medium, provided the original work is properly cited.

\begin{abstract}
This work proposes a convex functional endowed with a minimum, which occurs for the solution of the thermal radiation and natural convection heat transfer problem in a rectangular profile porous fin with a fluid flowing through it. The minimum principle ensures the (mathematically demonstrated) uniqueness of the solution and allows the problem simulation by employing a minimization procedure. Darcy's law with the Oberbeck-Boussinesq approximation simplifies the momentum equation. The energy equation assumes thermal equilibrium between the porous matrix and fluid, allowing comparisons with previous authors' models, which accounts for the effects of a porosity parameter, a radiation parameter, and a temperature ratio on the temperature. Results for very long fin and finite-length fin with insulated tip were successfully compared with previous works. Closed-form exact solutions for two limiting cases (no convection and no thermal radiation) are also presented.
\end{abstract}

\section{Introduction}

In many engineering applications, it is essential to improve the heat transfer rate between a surface and the surrounding fluid. Convection heat transfer between a given surface and its vicinity is enhanced if fins (characterized by high heat transfer rate with compact size and low cost) are connected to the surface. Also, thermal radiation is not negligible unless convection is substantially high. When natural convection is concerned, in many cases, thermal radiation cannot be disregarded.

The heat transfer enhancement could be significantly enlarged by employing porous fins, as introduced in the work of Kiwan and Al-Nimr [1]. These authors used a Darcy-Brinkman-Forchheimer model to compute the natural convection heat transfer from an array of porous fins attached to a hot surface and compared the results with those from an equal size arrangement with solid fins, finding up to $100 \%$ weight reduction of fin material for the same solid fins performance. The heat transfer enhancement with porous fins is particularly important when Darcy and Rayleigh numbers are high. Hamdan and AlNimr [2] later used the Darcy-Brinkman-Forchheimer model to compute the forced convection numerically when high-thermal conductivity porous fins are attached to the inner walls of two parallel-plate channels to enhance the heat transfer characteristics of the flow. They evaluated the effects of porous fin thickness, Darcy number, thermal conductivity ratio (ratio of porous thermal conductivity to fluid thermal conductivity), Reynolds number, and a microscopic inertial coefficient on the thermal performance. 
Kiwan [3] studied natural convection heat transfer from a rectangular porous fin, considering long fins, finite-length fins with insulated tip, and finite-length fins with tip subjected to a convection boundary condition, using Darcy's law as the momentum equation. He proposes a porous parameter $S_{H}$ relating Rayleigh and Darcy numbers, the thermal conductivity ratio, and a squared length/thickness ratio, which groups the geometric and flow parameters that influence the solution of the problem. However, increasing $S_{H}$ by increasing Rayleigh or Darcy numbers increases the heat transfer from the fin, but there is a limit for the increase of both the thermal conductivity ratio and the length/ thickness ratio, with the heat transfer rate reaching maximum values. Kiwan [4] also used Darcy's law as the momentum equation and the Rosseland approximation for a thermal radiation heat transfer conjugated with conduction to investigate the effect of radiation and natural convection heat transfer from a porous fin, so that the model is reduced to a single nonlinear ordinary differential equation, considering heat transfer within the porous media and between the porous media and the surroundings.

Several authors were concerned with the determination of the performance and optimum dimensions of porous fins. For instance, Kundu and Bhanja [5] proposed an approximate analytical methodology based on the Adomian decomposition to determine the performance and optimum dimensions of rectangular porous fins with consideration of three different models of predictions, together with the optimum porous fin design analysis. They considered governing equations previously used by Kiwan [3, 4] with one model considering convection heat transfer solely and a second one also accounting for thermal radiation, but assuming small temperature differences within the flow so that the radiation term can be linearized. Finally, the third model accounts for the real radiation term, giving rise to a highly nonlinear equation. Distinct porous fin shapes were subsequently considered $[6,7]$, and in Kundu and Lee [8], a variable temperature-dependent heat transfer coefficient was considered, along with convection and thermal radiation heat transfer. These authors developed an analytical formulation based on the calculus of variation, aiming at determining the minimum shape of porous fins.

$\mathrm{Ma}$ et al. [9] predicted the thermal performance of a porous fin considering natural convection and thermal radiation heat transfer, a temperature-dependent heat transfer coefficient, surface emissivity, and heat generation, using a spectral collocation method with a Lagrange polynomial interpolation, validating their results using both finite volumes homotopy perturbation methods. In subsequent work, $\mathrm{Ma}$ et al. [10] studied trapezoidal, convex parabolic, and concave parabolic profiles porous fins and combined conductive, convective, and radiative heat transfer, accounting for the temperature dependency and using a spectral element method, in which the domain was decomposed using a finite element strategy. Chebyshev polynomials were used to establish basis functions on each element.

The addition of a fin or an array of fins to the cavity walls may increase the overall heat transfer rate in a cavity. Alshuraiaan and Khanafer [11] used a Darcy-Forchheimer model to study natural convection in a differentially heated cavity with two thin porous fins attached to the hot wall and bottom insulated and solved the equations, obtained by a volume-average technique for a two-dimensional geometry, by finite element formulation based on the Galerkin method of weighted residuals. Asl et al. [12] also employed a Darcy-Forchheimer model to model the natural convection in an inclined rectangular enclosure with several porous fins attached to the hot wall for relevant parameters, comparing the enclosures with porous fins with both cavities with solid fins and/or cavities without fins. The governing equations for the fins were obtained using a volume-average technique and simulated by a finite volume method based on the SIMPLE method.

Gorla and Bakier [13] studied the effects of radiation and natural convection heat transfer in a rectangular profile porous fin, though which the fluid flows. The momentum equation is simplified by Darcy's model, and the energy equation assumes local thermal equilibrium and temperature variation only along the fin length and neglects radiant surface exchange. Three classical fins boundary conditions are considered: very long fin, insulated tip, and convective tip. The authors investigated the effects of a porosity parameter $S_{H}$ (relating Rayleigh and Darcy numbers, a thermal conductivity ratio, and the squared ratio length/thickness of the fin), a radiation parameter $G$ (relating the porous fin emissivity, Stefan-Boltzmann constant effective conductivity, the squared fin length, its thickness, and a temperature ratio and the difference between the base temperature and the ambient temperature elevated to the third power), and a temperature ratio $C_{T}$ (dimensionless ratio of ambient temperature and the difference between the base temperature and the ambient temperature) on the temperature distribution and heat transfer. They approximated the resulting nonlinear second-order ordinary differential equation using a fourth-order Runge-Kutta method. The authors observed that increasing $S_{H}$ by increasing either Rayleigh or Darcy numbers increases the heat transfer from fin; the $C_{T}$ parameter has a small influence on heat transfer while increasing the radiation parameter $G$ also increases heat transfer from fin for small values of Biot numbers. Torab and Yaghoobi [14] employed the same mechanical model used by [13] to analyze the rectangular porous fin but solved the highly nonlinear partial differential resulting equation using an analytical model: the differential transformation method based on a Taylor series expansion, obtaining good agreement with the results of [13]. Darvishi et al. [15] analyzed natural convection and radiation heat transfer in thoroughly wet porous fins with a model that can be considered as an extension of Gorla and Bakier [13] model. They analyzed the behavior of a porosity parameter $S_{H}$, a radiation parameter $G$, a temperature ratio $C_{T}$, and a wet fin parameter $m_{2}$ on the dimensionless temperature distribution and heat transfer rate. The highly nonlinear differential equation is solved numerically by using a spectral collocation method, after which they are reduced into algebraic equations using Chebyshev polynomials. Darvishi et al. [16] also considered thermal radiation and convection heat transfer, but in a rectangular radial porous fin, which is 
used in various applications and the ambient temperature effect is characterized differently from Gorla and Bakier [13]. The nonlinear equation is solved by employing a spectral collocation method with the unknown function approximated as a truncated series of Chebyshev polynomials. The results suggest that the radiation transfers more heat than a similar model without radiation.

A similar approach, but employing a systematic methodology, is used in the present work. The same mechanical model used by Gorla and Bakier [13] is considered. A convex functional is proposed to represent it, and its minimum is the solution to problem. A complete uniqueness proof is presented. Essentially, in this work, alternative modeling is proposed, which preserves the physical idea presented by Gorla and Bakier [13] but provides a considerable mathematical improvement. This improvement is represented by a minimum principle, characterized by a convex functional, which ensures the uniqueness of the solution and allows the problem to be simulated employing a minimization, instead of a standard discretization protocol. Also, it is important to note that the uniqueness of the solution is mathematically proved.

Besides, two limiting cases for infinite porous fins, namely, absence of thermal radiation and absence of convection (with the fin surrounded by an atmosphere-free space), have closedform exact solutions, which are presented.

\section{Mechanical Model}

A rectangular porous fin with constant cross section area and length $L$, width $W$, and thickness $t$, considered by Gorla and Bakier [13], allowing the flow to infiltrate through it, is considered. The porous matrix is assumed isotropic, homogeneous, and saturated by a single-phase fluid. Fluid and porous matrix (with constant physical properties) are assumed in thermal equilibrium, radiant interaction between the fin and its base is negligible, and the temperature does not vary across the fin thickness. The momentum equation is simplified by Darcy's law with Oberbeck-Boussinesq approximation [17]. Supposing temperature variation solely along the fin length and all the previous assumptions, the scaled energy balance can be expressed by the following onedimensional nonlinear equation $[13,14]$ :

$$
\begin{aligned}
\frac{\mathrm{d}^{2} \theta}{\mathrm{d} X^{2}}-S_{H} \theta^{2}-G\left[\left(\theta+C_{T}\right)^{4}-C_{T}^{4}\right] & =0, \\
\theta & =\frac{T-T_{\infty}}{T_{b}-T_{\infty}}, \\
X & =\frac{x}{L}, \\
S_{H} & =\frac{\mathrm{Da} \times \mathrm{Ra}}{k_{r}}\left(\frac{L}{t}\right)^{2}, \\
G & =\frac{2 \sigma \varepsilon}{k_{\mathrm{eff}} t} L^{2}\left(T_{b}-T_{\infty}\right)^{3}, \\
C_{T} & =\frac{T_{\infty}}{T_{b}-T_{\infty}},
\end{aligned}
$$

where $\theta$ represents the scaled temperature, $T_{b}$ is the temperature at the fin base and $T_{\infty}$ the environment temperature, $X$ is the dimensionless axial coordinate, $S_{H}$ is a porous parameter (accounting for permeability and buoyancy effects), $k_{\text {eff }}=(1-\varphi) k_{s}+\varphi k_{f}$ is the porous medium effective thermal conductivity, with $k_{s}$ being the porous matrix thermal conductivity (solid) and $k_{f}$ the fluid thermal conductivity, the conductivity ratio is given by $k_{r}=\left(k_{\mathrm{eff}} / k_{f}\right)$, and $\mathrm{Da}$ and Ra are Darcy and Rayleigh numbers given by

$$
\begin{aligned}
& \mathrm{Da}=\frac{K}{t^{2}}, \\
& \mathrm{Ra}=\frac{g \beta}{k_{f} v}\left(T_{b}-T_{\infty}\right) t^{3},
\end{aligned}
$$

with $K$ representing the porous matrix specific permeability, $g$ the gravitational acceleration, $\beta$ the thermal expansion coefficient, and $v$ the kinematic viscosity.

Besides, $G$ is a radiation parameter (indicating the effect of the fin surface emissivity) with $\sigma$ being the Stefan-Boltzmann constant and $\varepsilon$ the porous fin emissivity. The radiation model is based on the gray body assumption $[18,19]$. The $G$ parameter depends directly on the Stefan-Boltzmann constant and the emissivity. Moreover, as it may be noted, the increase in $G$ generates an increase in heat loss from radiation. Finally, $C_{T}$ is a temperature ratio.

The model presented in (1) is based on a previous work by Kiwan [3], in which he used Darcy's law with Oberbeck-Boussinesq approximation in the energy equation to describe the natural convection in a rectangular porous fin, proposing the porous parameter $S_{H}$ to describe the thermal performance of a porous fin. In a subsequent work [4], Kiwan accounted for thermal radiation heat transfer. The radial porous fin with variable thermal conductivity [16] could be reduced to model (1) if the thermal conductivity was supposed constant, and the fin was rectangular. Besides, if the moving porous fin [20] were made stationary under a steady-state regimen, or in a fully wet porous fin [15] if the wet fin parameter were made zero, model (1) would be recovered. The exact model (1) is employed in $[21,22]$.

\section{A Convex Functional with Minimum Principle}

3.1. Insulated Tip Porous Fin. Considering (1), a porous fin with insulated tip is represented by

$$
\frac{\mathrm{d}^{2} \theta}{\mathrm{d} X^{2}}-S_{H} \theta^{2}-G\left[\left(\theta+C_{T}\right)^{4}-C_{T}^{4}\right]=0\left\{\begin{array}{l}
\theta(0)=1, \\
\left.\frac{\mathrm{d} \theta}{\mathrm{d} X}\right|_{X=1}=0 .
\end{array}\right.
$$

The following convex functional is proposed, such that its minimization will correspond to the solution of the problem (3): 
$I[w]=\int_{0}^{1}\left\{\frac{1}{2}\left(\frac{\mathrm{d} w}{\mathrm{~d} X}\right)^{2}+\frac{S_{H}}{3}|w|^{3}+\frac{G}{5}\left|w+C_{T}\right|^{5}-G C_{T}^{4} w\right\} \mathrm{d} X$ with $w=1$ for $X=0$.

In other words, the function $u$ such that, for any $w$, $I[w] \geq I[u]$, is the solution of problem (3). Since $u$ corresponds to an extremum of the functional $I[w]$, the first variation of $I[w]$ for $w \equiv u$ must be zero.

The first variation of the functional proposed in (4) is given by [23]

$$
\begin{aligned}
\delta I[w]= & \int_{0}^{1}\left\{\left(\frac{\mathrm{d} w}{\mathrm{~d} X}\right) \delta\left(\frac{\mathrm{d} w}{\mathrm{~d} X}\right)+S_{H}|w| w \delta w+G\left|w+C_{T}\right|^{3}\right. \\
& \left.\cdot\left(w+C_{T}\right) \delta w-G C_{T}^{4} \delta w\right\} \mathrm{d} X, \text { with } \delta w=0 \text { for } X=0,
\end{aligned}
$$

where

$$
\begin{aligned}
\int_{0}^{1}\left(\frac{\mathrm{d} w}{\mathrm{~d} X}\right) \delta\left(\frac{\mathrm{d} w}{\mathrm{~d} X}\right) \mathrm{d} X & =\int_{0}^{1}\left(\frac{\mathrm{d} w}{\mathrm{~d} X}\right)\left(\frac{\mathrm{d} \delta w}{\mathrm{~d} X}\right) \mathrm{d} X \\
& =\int_{0}^{1}\left\{\frac{\mathrm{d}}{\mathrm{d} X}\left(\delta w \frac{\mathrm{d} w}{\mathrm{~d} X}\right)-\delta w\left(\frac{\mathrm{d}^{2} w}{\mathrm{~d} X^{2}}\right)\right\} \mathrm{d} X \\
& =-\int_{0}^{1} \delta w\left(\frac{\mathrm{d}^{2} w}{\mathrm{~d} X^{2}}\right) \mathrm{d} X+\left(\delta w \frac{\mathrm{d} w}{\mathrm{~d} X}\right)_{0}^{1} \\
& =-\int_{0}^{1} \delta w\left(\frac{\mathrm{d}^{2} w}{\mathrm{~d} X^{2}}\right) \mathrm{d} X+\left(\frac{\mathrm{d} w}{\mathrm{~d} X}\right)_{X=1} \delta w
\end{aligned}
$$

$$
\begin{aligned}
\delta I= & \int_{0}^{1}\left\{-\frac{\mathrm{d}^{2} w}{\mathrm{~d} X^{2}} \delta w+S_{H}|w| w \delta w+G\left|w+C_{T}\right|^{3}\right. \\
& \left.\cdot\left(w+C_{T}\right) \delta w-G C_{T}^{4} \delta w\right\} \mathrm{d} X+\left.\frac{\mathrm{d} w}{\mathrm{~d} X}\right|_{X=1} \delta w .
\end{aligned}
$$

To ensure that the first variation is zero $(\delta I=0)$, the Euler-Lagrange equation and the natural boundary condition at $X=1$ must be satisfied. The function $u$ that makes $\delta I=0$ is the solution of the following equation:

$$
\begin{aligned}
& -\frac{\mathrm{d}^{2} u}{\mathrm{~d} X^{2}}+S_{H}|u| u+G\left|u+C_{T}\right|^{3}\left(u+C_{T}\right) \\
& -G C_{T}^{4}=0, \quad 0<X<1 \text { (Euler-Lagrange equation), }
\end{aligned}
$$

$\left.\frac{\mathrm{d} u}{\mathrm{~d} X}\right|_{X=1}=0$ (natural boundary condition).

Since $\theta$ is a nonnegative valued function, (8) corresponds precisely to (3), the original problem.

The next step is to show that the proposed functional (4) is strictly convex, ensuring that $\delta I=0$ corresponds to a unique minimum. In other words, the solution's uniqueness is ensured.

At this point, it will be demonstrated that the functional proposed in (4) is strictly convex, thus ensuring that $\delta I=0$ corresponds to a unique minimum value. If $(1 / 2)(\mathrm{d} w / \mathrm{d} X)^{2}$ is shown to be convex and both $\left(S_{H} / 3\right)|w|^{3}$ and $(G / 5)\left|w+C_{T}\right|^{5}$ are proven to be strictly convex, the functional proposed in (4) is strictly convex, since the linear term has no impact on the convexity [23].

For convenience, let us introduce the functions $u_{1}$ and $u_{2}$, such that $u_{1} \neq u_{2}$, and the parameter $\phi \in(0,1)$. Now, take into account that

Substituting the above identity in the first variation (in (5)),

$$
\begin{aligned}
&\left(\frac{\mathrm{d}}{\mathrm{d} X}\left(\phi u_{1}+(1-\phi) u_{2}\right)\right)^{2} \leq \phi\left(\frac{\mathrm{d} u_{1}}{\mathrm{~d} X}\right)^{2}+(1-\phi)\left(\frac{\mathrm{d} u_{2}}{\mathrm{~d} X}\right)^{2},\left(\phi \frac{\mathrm{d} u_{1}}{\mathrm{~d} X}+(1-\phi) \frac{\mathrm{d} u_{2}}{\mathrm{~d} X}\right)^{2} \leq \phi\left(\frac{\mathrm{d} u_{1}}{\mathrm{~d} X}\right)^{2}+(1-\phi)\left(\frac{\mathrm{d} u_{2}}{\mathrm{~d} X}\right)^{2}, \\
& \phi^{2}\left(\frac{\mathrm{d} u_{1}}{\mathrm{~d} X}\right)^{2}+(1-\phi)^{2}\left(\frac{\mathrm{d} u_{2}}{\mathrm{~d} X}\right)^{2}+2 \phi(1-\phi)\left(\frac{\mathrm{d} u_{1}}{\mathrm{~d} X}\right)\left(\frac{\mathrm{d} u_{2}}{\mathrm{~d} X}\right) \leq \phi\left(\frac{\mathrm{d} u_{1}}{\mathrm{~d} X}\right)^{2}+(1-\phi)\left(\frac{\mathrm{d} u_{2}}{\mathrm{~d} X}\right)^{2}, \\
& 2 \phi(1-\phi)\left(\frac{\mathrm{d} u_{1}}{\mathrm{~d} X}\right)\left(\frac{\mathrm{d} u_{2}}{\mathrm{~d} X}\right) \leq \phi(1-\phi)\left(\frac{\mathrm{d} u_{1}}{\mathrm{~d} X}\right)^{2}+\phi(1-\phi)\left(\frac{\mathrm{d} u_{2}}{\mathrm{~d} X}\right)^{2}, \\
& 2\left(\frac{\mathrm{d} u_{1}}{\mathrm{~d} X}\right)\left(\frac{\mathrm{d} u_{2}}{\mathrm{~d} X}\right) \leq\left(\frac{\mathrm{d} u_{1}}{\mathrm{~d} X}\right)^{2}+\left(\frac{\mathrm{d} u_{2}}{\mathrm{~d} X}\right)^{2} \Rightarrow\left(\frac{\mathrm{d} u_{1}}{\mathrm{~d} X}-\frac{\mathrm{d} u_{2}}{\mathrm{~d} X}\right)^{2} \geq 0 .
\end{aligned}
$$


Equation (9) allows concluding that $(1 / 2)(\mathrm{d} w / \mathrm{d} X)^{2}$ is convex (not strictly convex, because $w$ could be a constant).

Since the second derivative (with respect to $w$ ) is given by

$$
\frac{\mathrm{d}^{2}}{\mathrm{~d} w^{2}}\left[\frac{S_{H}}{3}|w|^{3}+\frac{G}{5}\left|w+C_{T}\right|^{5}\right]=2 S_{H}|w|+4 G\left|w+C_{T}\right|^{3}
$$

and since it, consequently, is always positive valued, it is ensured that the sum $\left(S_{H} / 3\right)|w|^{3}+(G / 5)\left|w+C_{T}\right|^{5}$ is strictly convex. Therefore, the minimum of the functional is unique, ensuring the uniqueness of the solution.

At this point, it may be stated that (4) represents a minimum principle.

3.2. Porous Fin with Prescribed Convection at the Tip. Consider (1) with prescribed convection at the fin extremity, represented by

$$
\begin{aligned}
& \frac{\mathrm{d}^{2} \theta}{\mathrm{d} X^{2}}-S_{H} \theta^{2}-G\left[\left(\theta+C_{T}\right)^{4}-C_{T}^{4}\right] \\
& \quad=0,\left\{\begin{array}{l}
\theta(0)=1, \\
\left.\frac{\mathrm{d} \theta}{\mathrm{d} X}\right|_{X=1}+B i \theta(1)=0 .
\end{array}\right.
\end{aligned}
$$

The following convex functional is proposed, such that its minimization will correspond to the solution of problem (11):

$$
\begin{aligned}
I[w]= & \int_{0}^{1}\left\{\frac{1}{2}\left(\frac{\mathrm{d} w}{\mathrm{~d} X}\right)^{2}+\frac{S_{H}}{3}|w|^{3}+\frac{G}{5}\left|w+C_{T}\right|^{5}\right. \\
& \left.-G C_{T}^{4} w\right\} \mathrm{d} X+\frac{1}{2} \operatorname{Bi}\left[w^{2}\right]_{X=1}, \text { with } w=1 \text { for } X=0 .
\end{aligned}
$$

The first variation of the functional proposed in (12) is given by [23] (Appendix A)

$$
\begin{aligned}
\delta I= & \int_{0}^{1}\left\{\left(\frac{\mathrm{d} w}{\mathrm{~d} X}\right) \delta\left(\frac{\mathrm{d} w}{\mathrm{~d} X}\right)+S_{H}|w| w \delta w+G\left|w+C_{T}\right|^{3}\right. \\
& \left.\cdot\left(w+C_{T}\right) \delta w-G C_{T}^{4} \delta w\right\} \mathrm{d} X+B i[w \delta w]_{X=1}, \text { with } \delta w \\
= & 0 \text { for } X=0,
\end{aligned}
$$

where

$$
\begin{aligned}
\int_{0}^{1}\left(\frac{\mathrm{d} w}{\mathrm{~d} X}\right) \delta\left(\frac{\mathrm{d} w}{\mathrm{~d} X}\right) \mathrm{d} X & =\int_{0}^{1}\left(\frac{\mathrm{d} w}{\mathrm{~d} X}\right)\left(\frac{\mathrm{d} \delta w}{\mathrm{~d} X}\right) \mathrm{d} X \\
& =\int_{0}^{1}\left\{\left(\frac{\mathrm{d}}{\mathrm{d} X}\right)\left(\delta w \frac{\mathrm{d} w}{\mathrm{~d} X}\right)-\delta w\left(\frac{\mathrm{d}^{2} w}{\mathrm{~d} X^{2}}\right)\right\} \mathrm{d} X \\
& =-\int_{0}^{1} \delta w\left(\frac{\mathrm{d}^{2} w}{\mathrm{~d} X^{2}}\right) \mathrm{d} X+\left(\delta w \frac{\mathrm{d} w}{\mathrm{~d} X}\right)_{0}^{1} \\
& =-\int_{0}^{1} \delta w\left(\frac{\mathrm{d}^{2} w}{\mathrm{~d} X^{2}}\right) \mathrm{d} X+\left(\frac{\mathrm{d} w}{\mathrm{~d} X}\right)_{X=1}[\delta w]_{X=1}
\end{aligned}
$$

So, the first variation can be expressed as

$$
\begin{aligned}
\delta I= & \int_{0}^{1}\left\{-\left(\frac{\mathrm{d}^{2} w}{\mathrm{~d} X^{2}}\right) \delta w+S_{H}|w| w \delta w+G\left|w+C_{T}\right|^{3}\right. \\
& \left.\cdot\left(w+C_{T}\right) \delta w-G C_{T}^{4} \delta w\right\} \mathrm{d} X+\left\{\left(\frac{\mathrm{d} w}{\mathrm{~d} X}\right)_{X=1}\right. \\
& \left.+\operatorname{Bi}[w]_{X=1}\right\}[\delta w]_{X=1} .
\end{aligned}
$$

To ensure that $\delta I=0$, the Euler-Lagrange equation and the natural boundary condition at $X=1$ must be fulfilled. The function $u$ that makes $\delta I=0$ is the solution of

$$
\begin{array}{r}
-\frac{\mathrm{d}^{2} u}{\mathrm{~d} X^{2}}+S_{H}|u| u+G\left|u+C_{T}\right|^{3}\left(u+C_{T}\right)-G C_{T}^{4}=0, \\
0<X<1 ;\left(\frac{\mathrm{d} u}{\mathrm{~d} X}\right)_{X=1}+\operatorname{Bi}[u]_{X=1}=0 .
\end{array}
$$

Equation (16) corresponds, exactly, to the original equation (11), which is the original problem.

\section{Results}

The minimization of $I[w]$ was performed with the aid of a conjugate gradient method [24, 25]. Essentially, in this methodology, the search directions are built by conjugation of the residuals. These residuals, in turn, are orthogonal to all the previous residuals and exploration directions. This property leads to a new, linearly independent, exploration direction, whenever the residuals are nonzero. Repeating the procedure, the solution is reached. The minimization of the functional, in all cases, was carried out considering a continuous piecewise linear approximation given by

$$
\begin{aligned}
w & =w_{i}+\frac{w_{i+1}-w_{i}}{\Delta x}\left(x-x_{i}\right), \quad x_{i}<x<x_{i+1}, i=1,2, \ldots, N \\
\Delta x & =\frac{L}{N-1} ; x_{i}=(i-1) \Delta x,
\end{aligned}
$$

in which (always) $w_{1}=1$.

The results were obtained with $N=1001$. When $N>500$, no improvement in the results can be observed.

Aiming at showing the excellent performance of minimization of the proposed convex functionals, results for distinct values of the behavior of a porosity parameter $S_{H}$, a radiation parameter $G$, and a temperature ratio $C_{T}$, considering both insulated tip and long porous fins on the temperature distribution, are compared with those from Gorla and Bakier [13], as depicted in Figures 1-3. In all cases, results from the present work are represented by lines with symbols while lines without symbols represent results from [13]. Figure 1 considers the effect of a porosity parameter $S_{H}$ on the temperature distribution along with the axial distance 
along the fin for both insulated tip porous fin and long porous fin, for $S_{H}=1.0, S_{H}=10.0$, and $S_{H}=100.0$, fixing $G=0.1$ and $C_{T}=0.1$. As can be noted, all the results obtained with the proposed methodology show perfect agreement with [13].

It is important to note that porosity alters the permeability and the effective thermal conductivity of the porous fin, consequently affecting the $S_{H}$ coefficient. This coefficient, in turn, directly impacts the effectiveness of heat exchange by convection.

Figure 1 shows that as the porous parameter increases, convection heat transfer also increases, while radiation heat transfer is maintained small. So, convection is the dominant heat transfer mechanism in the cases considered in this figure.

In Figure 2, the effect of a radiation parameter $G$ (considering $G=0.1, G=1.0$, and $G=10.0$ ) on the temperature distribution along the axial distance along the fin is successfully compared with [13] for both long fin and insulated tip porous fin. The parameters $S_{H}=1.0$ and $C_{T}=0.1$ were maintained constant to allow the comparison.

It is important to note that as the radiation parameter $\mathrm{G}$ increases, the heat transfer also increases. Besides the convection heat transfer loss, there is also radiation heat transfer loss in the porous fin.

In Figure 2, the dominant heat transfer mechanism is thermal radiation heat transfer, since the porous parameter (and, consequently, convection heat transfer) is kept small in the cases depicted in this figure.

Finally, Figure 3 depicts the effective comparison with [13] of distinct values of temperature ratios $C_{T}, C_{T}=0.01$, $C_{T}=0.1$, and $C_{T}=0.5$ (also including the results for $\left.C_{T}=1.0\right)$ for an insulated tip and long porous fins, considering $G=0.1$ and $S_{H}=1.0$.

In Figure 3, both convection and radiation play a significant role. It is convenient for evaluating the temperature ratios' effect properly. This effect is more critical for insulated tip porous fins.

Since $C_{T}$ is nonnegative valued, an increase of $C_{T}$ gives rise to an increase in the radiation heat loss. It is easy to see this from the following relation:

$$
G\left(\left(\theta+C_{T}\right)^{4}-C_{T}^{4}\right)=G\left(\theta^{4}+4 \theta^{3} C_{T}+6 \theta^{2} C_{T}^{2}+4 \theta C_{T}^{3}\right) .
$$

Figure 4 shows the first derivative of the scaled temperature at zero for distinct values of a porosity parameter $S_{H}$, a radiation parameter $G$, and a temperature ratio $C_{T}$.

In Table 1 , the temperature gradient $\left(-\theta^{\prime}(0)\right)$ is successfully compared for all values considered in [13]. Some additional values are also considered. The results depicted in Table 1 are also in good agreement with those obtained by Torab and Yaghoobi [14], which, in turn, agree with [13].

Comparing the values obtained for $-\theta^{\prime}(0)$, acquired with several values of $C_{T}, G$, and $S_{H}$, it may be noted that an increase of $C_{T}$ impacts $-\theta^{\prime}(0)$ only for high values of $G$. On the other hand, for values of $S_{H}$ such that $S_{H} \geq 100 G$, the quantity $-\theta^{\prime}(0)$ is slightly affected by the radiation heat transfer, independently of $C_{T}$.

It is important to remark the robustness of the proposed methodology, which allows considering other combinations of the parameters-namely, the porosity parameter, the radiation parameter, and the temperature ratio.

\section{Two Limiting Cases with Closed-Form Exact Solutions}

When there is no radiation heat transfer, which means $G=0$, (1) reduces to

$$
\frac{\mathrm{d}^{2} \theta}{\mathrm{d} X^{2}}-S_{H} \theta^{2}=0, \quad \theta(0)=1, \theta(\infty)=0 .
$$

In this case, for an infinite fin, the following closed-form exact solution can be obtained:

$$
\theta=\left(\frac{\sqrt{6}}{\left(\sqrt{S_{H}}\right) x+\sqrt{6}}\right)^{2}
$$

that gives rise to the following result for $\theta^{\prime}(0)$ :

$$
\theta^{\prime}(0)=-\sqrt{\frac{2 S_{H}}{3}} \text {. }
$$

When there is no convection and an atmosphere-free space surrounds the fin (a case in which $C_{T}$ and $S_{H}$ are equal to zero), (1) reduces to

$$
\frac{\mathrm{d}^{2} \theta}{\mathrm{d} X^{2}}-G \theta^{4}=0, \quad \theta(0)=1, \theta(\infty)=0 .
$$

In this case, the following closed-form exact solution is obtained for an infinite fin:

$$
\theta=\left(\frac{\sqrt{10}}{3(\sqrt{G}) x+\sqrt{10}}\right)^{2 / 3},
$$

giving rise to the following result for $\theta^{\prime}(0)$ :

$$
\theta^{\prime}(0)=-\sqrt{\frac{2 G}{5}}
$$

\section{Final Remarks}

It is important to note that whenever the scaled temperature $\theta$ is positive, the solution coincides with the equation proposed in [13]. Besides, the temperature gradient has shown good agreement with [13].

The alternative model proposed in this work-a convex functional with a minimum principle-allows the problem to be simulated by minimizing the functional, ensuring the 

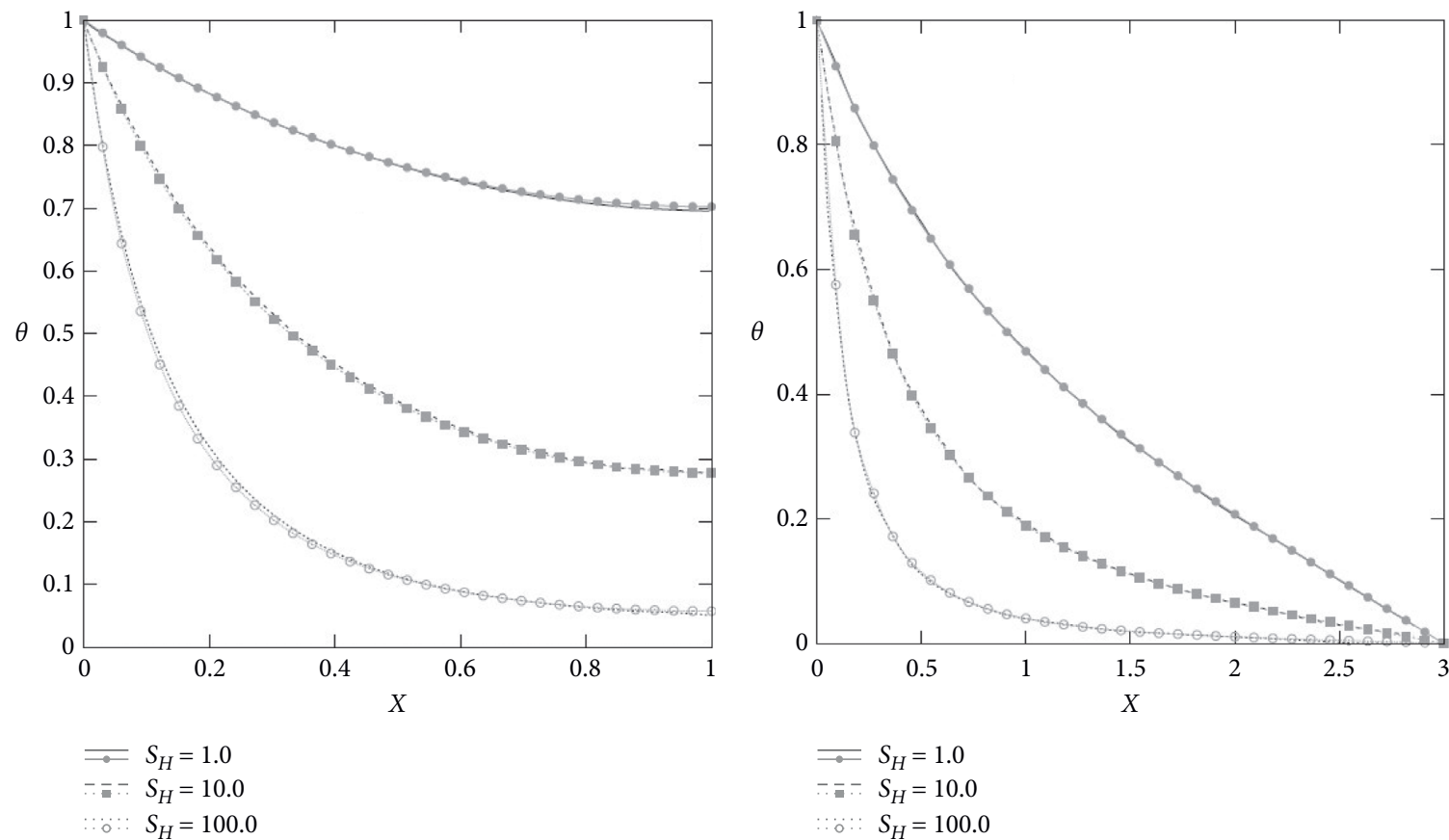

(a)

(b)

FIGURE 1: Effect of a porosity parameter on the temperature distribution: (a) insulated tip porous fin and (b) very long porous fin.

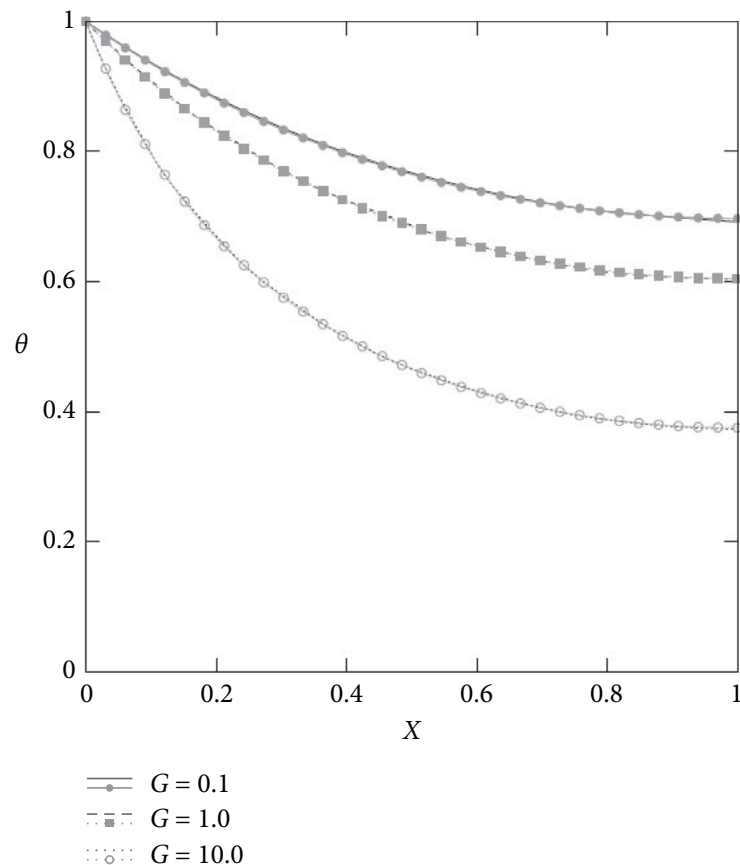

(a)

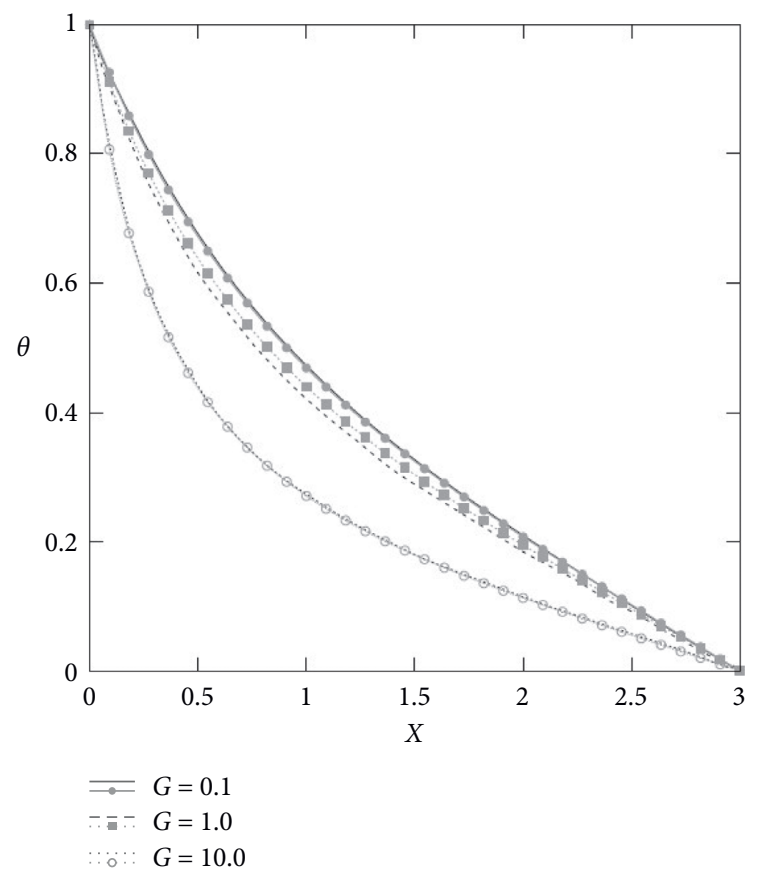

(b)

FIGURE 2: Effect of a radiation parameter on the temperature distribution: (a) insulated tip porous fin and (b) very long porous fin.

uniqueness of the solution and many possible combinations of the parameters $S_{H}, G$, and $C_{T}$, due to the model robustness.
Besides, the exact closed-form solution for the limiting cases allows the exact computation of $\theta^{\prime}(0)$ for both limiting cases. 


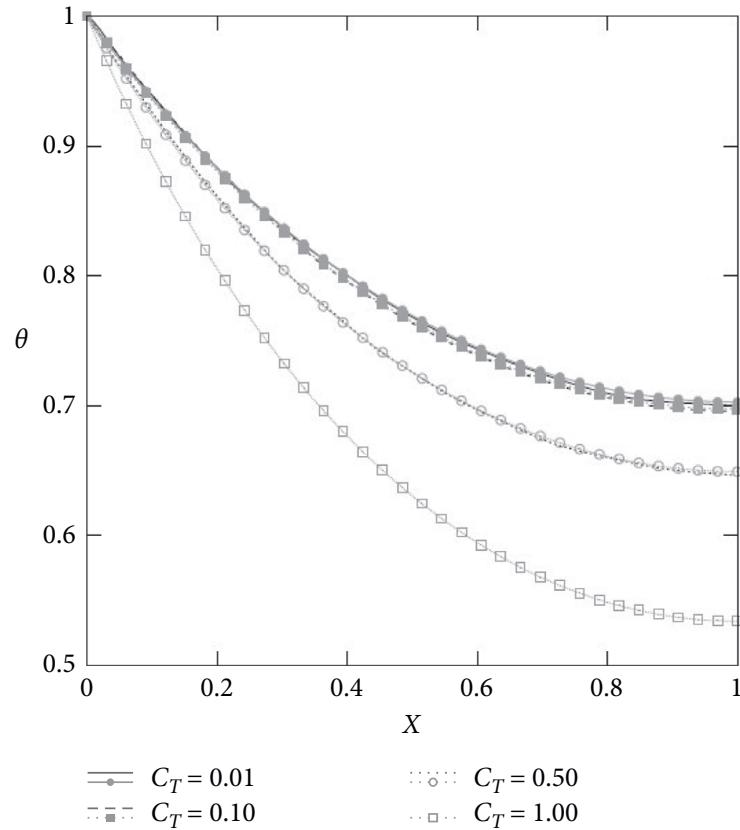

(a)

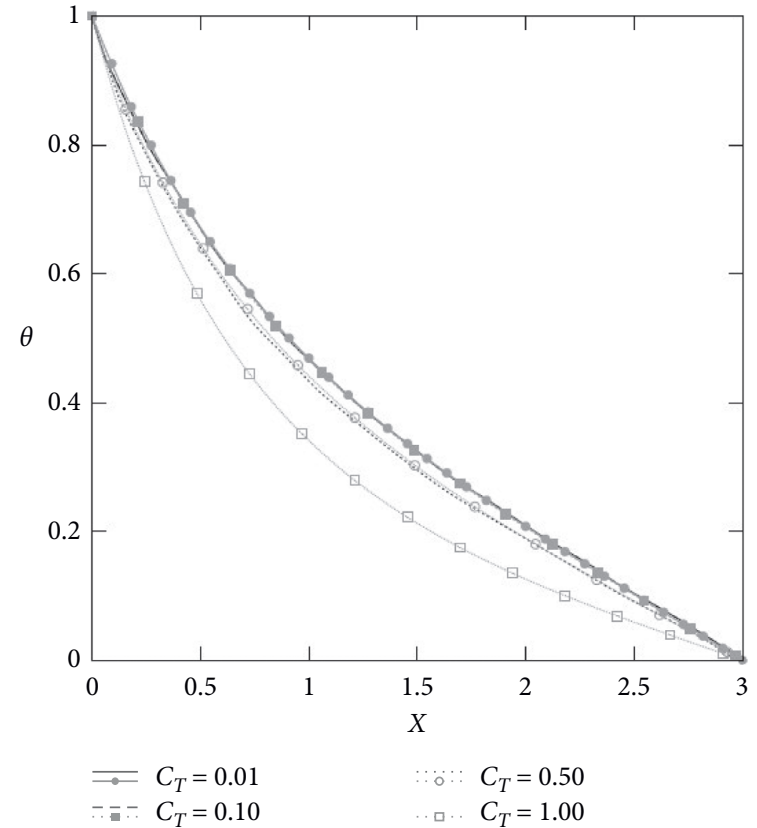

(b)

Figure 3: Effect of a temperature ratio on the temperature distribution: (a) insulated tip porous fin and (b) very long porous fin.

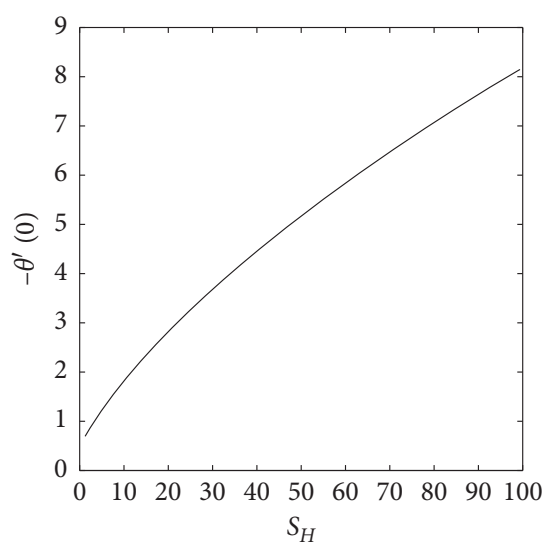

(a)

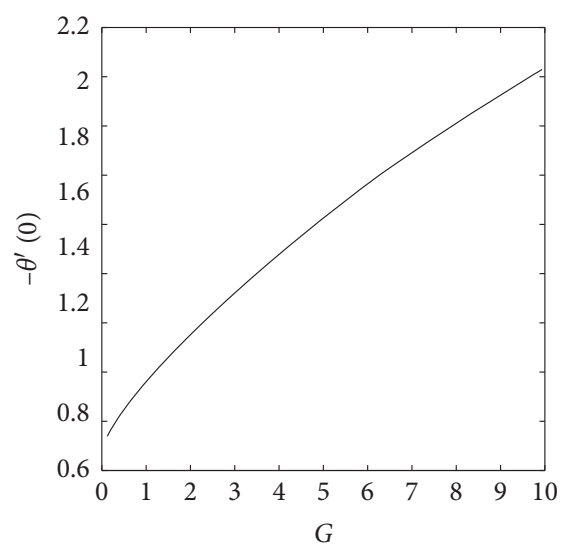

(b)

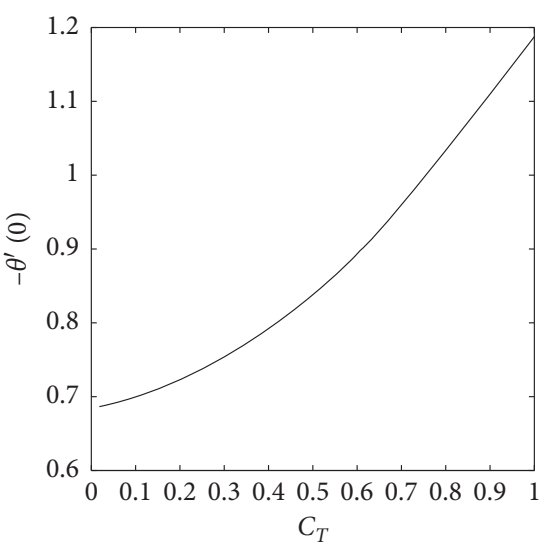

(c)

Figure 4: $-\theta^{\prime}(0)$ versus (a) porosity parameter $S_{H}\left(G=0.1\right.$ and $\left.C_{T}=0.1\right)$, (b) radiation parameter $G\left(S_{H}=1.0\right.$ and $\left.C_{T}=0.1\right)$, and (c) temperature ratio $C_{T}\left(G=0.1\right.$ and $\left.S_{H}=1.0\right)$.

\section{Appendix}

\section{A. On the First Variation of the Functional Defined in (4)}

Let us consider the functional $I[w]$ that reaches an extremum when $w \equiv u$. For any admissible function $\eta$ and any real-valued parameter $\varepsilon$, we have that this extremum is such that

$$
\frac{\mathrm{d}}{\mathrm{d} \varepsilon}\{I[u+\varepsilon \eta]\}_{\varepsilon=0}=0
$$

We define the first variation of $I[w]$ as follows:

$$
\delta I=\frac{\mathrm{d}}{\mathrm{d} \varepsilon}\{I[u+\varepsilon \eta]\} .
$$

If this extremum corresponds to a minimum (which is the case considered here, since the functional is strictly convex), we have

$$
I[w]=I[u+\varepsilon \eta]>I[u], \quad \varepsilon \eta \neq 0 .
$$

The first variation of a functional is given by its first derivative with respect to $\varepsilon$. So, 
TABLE 1: Temperature gradient $-\theta^{\prime}(0)$ for distinct values of $S_{H}, G$, and $C_{T}$ computed in the present paper [*] and by Gorla and Bakier [13] for insulated fin and very long fin.

\begin{tabular}{|c|c|c|c|c|c|c|}
\hline$S_{H}$ & $G$ & $C_{T}$ & $-\theta^{\prime}(0)\left[{ }^{*}\right]$ insulated & $-\theta^{\prime}(0)[13]$ insulated & $-\theta^{\prime}(0)\left[{ }^{*}\right]$ long & $-\theta^{\prime}(0)$ [13] long \\
\hline 1.00 & 10.0 & 0.01 & 2.17619 & 2.1763 & 2.21137 & 2.2111 \\
\hline 1.00 & 10.0 & 0.10 & 2.64106 & 2.6413 & 2.66766 & 2.6678 \\
\hline 1.00 & 10.0 & 0.50 & 5.44088 & 5.4420 & 5.44419 & 5.4467 \\
\hline 1.00 & 10.0 & 1.00 & 10.22574 & & 10.21980 & \\
\hline 1.00 & 1.00 & 0.01 & 0.93334 & 0.9334 & 1.06124 & 1.0582 \\
\hline 1.00 & 1.00 & 0.10 & 1.04642 & 1.0464 & 1.15980 & 1.1574 \\
\hline 1.00 & 1.00 & 0.50 & 1.82689 & 1.8269 & 1.89033 & 1.8901 \\
\hline 1.00 & 1.00 & 1.00 & 3.30814 & & 3.32628 & \\
\hline 1.00 & 0.10 & 0.01 & 0.68614 & 0.6861 & 0.86847 & 0.8637 \\
\hline 1.00 & 0.10 & 0.10 & 0.70212 & 0.7021 & 0.88081 & 0.8762 \\
\hline 1.00 & 0.10 & 0.50 & 0.83892 & 0.8389 & 0.99593 & 0.9928 \\
\hline 1.00 & 0.10 & 1.00 & 1.18902 & & 1.31176 & \\
\hline 10.0 & 10.0 & 0.01 & 3.27878 & 3.2791 & 3.29690 & 3.2975 \\
\hline 10.0 & 10.0 & 0.10 & 3.60551 & 3.6059 & 3.61979 & 3.6206 \\
\hline 10.0 & 10.0 & 0.50 & 5.96756 & 5.9690 & 5.96904 & 5.9722 \\
\hline 10.0 & 10.0 & 1.00 & 10.51447 & & 10.50804 & \\
\hline 10.0 & 1.00 & 0.01 & 2.63499 & 2.6351 & 2.66264 & 2.6628 \\
\hline 10.0 & 1.00 & 0.10 & 2.67758 & 2.6777 & 2.70427 & 2.7044 \\
\hline 10.0 & 1.00 & 0.50 & 3.07271 & 3.0729 & 3.09290 & 3.0932 \\
\hline 10.0 & 1.00 & 1.00 & 4.12188 & & 4.13045 & \\
\hline 10.0 & 0.10 & 0.01 & 2.56154 & 2.5616 & 2.59067 & 2.5907 \\
\hline 10.0 & 0.10 & 0.10 & 2.56595 & 2.5660 & 2.59498 & 2.5951 \\
\hline 10.0 & 0.10 & 0.50 & 2.60992 & 2.6100 & 2.63801 & 2.6381 \\
\hline 10.0 & 0.10 & 1.00 & 2.75047 & & 2.77625 & \\
\hline 100.0 & 10.0 & 0.01 & 8.41470 & 8.4178 & 8.41173 & 8.4185 \\
\hline 100.0 & 10.0 & 0.10 & 8.54630 & 8.5496 & 8.54306 & 8.5506 \\
\hline 100.0 & 10.0 & 0.50 & 9.77565 & 9.7806 & 9.77013 & 9.7810 \\
\hline 100.0 & 10.0 & 1.00 & 13.05389 & & 13.04186 & \\
\hline 100.0 & 1.00 & 0.01 & 8.18740 & 8.1899 & 8.18510 & 8.1907 \\
\hline 100.0 & 1.00 & 0.10 & 8.20103 & 8.2036 & 8.19870 & 8.2043 \\
\hline 100.0 & 1.00 & 0.50 & 8.33727 & 8.3400 & 8.33474 & 8.3407 \\
\hline 100.0 & 1.00 & 1.00 & 8.77516 & & 8.77199 & \\
\hline 100.0 & 0.10 & 0.01 & 8.16432 & 8.1668 & 8.16209 & 8.1676 \\
\hline 100.0 & 0.10 & 0.10 & 8.16569 & 8.2036 & 8.17722 & 8.1689 \\
\hline 100.0 & 0.10 & 0.50 & 8.17948 & 8.1820 & 8.16159 & 8.1824 \\
\hline 100.0 & 0.10 & 1.00 & 8.22515 & & 8.20699 & \\
\hline 1000.0 & 1.00 & 0.01 & 25.57136 & & 25.81644 & \\
\hline
\end{tabular}

$$
\begin{aligned}
\delta I[w]= & \frac{\mathrm{d} I}{\mathrm{~d} \varepsilon}=\frac{\mathrm{d}}{\mathrm{d} \varepsilon}\left\{\int _ { 0 } ^ { 1 } \left\{\frac{1}{2}\left(\frac{\mathrm{d} w}{\mathrm{~d} X}\right)^{2}+\frac{S_{H}}{3}|w|^{3}+\frac{G}{5}\left|w+C_{T}\right|^{5}\right.\right. \\
& \left.\left.-G C_{T}^{4} w\right\} \mathrm{~d} X\right\}=\frac{\mathrm{d}}{\mathrm{d} \varepsilon}\left\{\int _ { 0 } ^ { 1 } \left\{\frac{1}{2}\left(\frac{\mathrm{d}}{\mathrm{d} X}(u+\varepsilon \eta)\right)^{2}\right.\right. \\
& \left.\left.+\frac{S_{H}}{3}|u+\varepsilon \eta|^{3}+\frac{G}{5}\left|u+\varepsilon \eta+C_{T}\right|^{5}-G C_{T}^{4}(u+\varepsilon \eta)\right\} \mathrm{d} X\right\},
\end{aligned}
$$

in which $\eta$ is called the first variation of $w$, which means the first derivative of $w$ with respect to $\varepsilon$. Therefore,

$$
\begin{aligned}
\delta I[w]= & \frac{\mathrm{d} I}{\mathrm{~d} \varepsilon}=\left\{\int _ { 0 } ^ { 1 } \left\{\left(\frac{\mathrm{d}}{\mathrm{d} X}(u+\varepsilon \eta)\right) \frac{\mathrm{d} \eta}{\mathrm{d} X}+S_{H}|u+\varepsilon \eta|\right.\right. \\
& \left.\left.\cdot(u+\varepsilon \eta) \eta+G\left|u+\varepsilon \eta+C_{T}\right|^{3}\left(u+\varepsilon \eta+C_{T}\right) \eta-G C_{T}^{4} \eta\right\} \mathrm{d} X\right\} \\
= & \int_{0}^{1}\left\{\left(\frac{\mathrm{d} w}{\mathrm{~d} X}\right)\left(\frac{\mathrm{d}(\delta w)}{\mathrm{d} X}\right)+S_{H}|w| w \delta w+G\left|w+C_{T}\right|^{3}\right. \\
& \left.\cdot\left(w+C_{T}\right) \delta w-G C_{T}^{4} \delta w\right\} \mathrm{d} X \\
= & \int_{0}^{1}\left\{\left(\frac{\mathrm{d} w}{\mathrm{~d} X}\right)\left(\delta \frac{\mathrm{d} w}{\mathrm{~d} X}\right)+S_{H}|w| w \delta w+G\left|w+C_{T}\right|^{3}\right. \\
& \left.\cdot\left(w+C_{T}\right) \delta w-G C_{T}^{4} \delta w\right\} \mathrm{d} X .
\end{aligned}
$$




\section{Data Availability}

The data used to support the findings of this study are available within the article.

\section{Conflicts of Interest}

The authors declare that there are no conflicts of interest regarding the publication of this paper.

\section{Acknowledgments}

The authors Maria Laura Martins-Costa and Rogério M. Saldanha da Gama gratefully acknowledge the financial support provided by the Brazilian agency $\mathrm{CNPq}$, while the author Vinícius Vendas Sarmento gratefully acknowledges the financial support provided by the Brazilian agency CAPES.

\section{References}

[1] S. Kiwan and M. A. Al-Nimr, "Using porous fins for heat transfer enhancement," Journal of Heat Transfer, vol. 123, no. 4, pp. 790-795, 2001.

[2] M. Hamdan and M. A. Al-Nimr, "The use of porous fins for heat transfer augmentation in parallel-plate channels," Transport in Porous Media, vol. 84, no. 2, pp. 409-420, 2010.

[3] S. Kiwan, "Thermal analysis of natural convection porous fins," Transport in Porous Media, vol. 67, no. 1, pp. 17-29, 2007.

[4] S. Kiwan, "Effect of radiative losses on the heat transfer from porous fins," International Journal of Thermal Sciences, vol. 46, no. 10, pp. 1046-1055, 2007.

[5] B. Kundu and D. Bhanja, "An analytical prediction for performance and optimum design analysis of porous fins," International Journal of Refrigeration, vol. 34, no. 1, pp. 337-352, 2011.

[6] B. Kundu, D. Bhanja, and K.-S. Lee, "A model on the basis of analytics for computing maximum heat transfer in porous fins," International Journal of Heat and Mass Transfer, vol. 55, no. 25-26, pp. 7611-7622, 2012.

[7] D. Bhanja and B. Kundu, "Thermal analysis of a constructal T-shaped porous fin with radiation effects," International Journal of Refrigeration, vol. 34, no. 6, pp. 1483-1496, 2011.

[8] B. Kundu and K.-S. Lee, "Exact analysis for minimum shape of porous fins under convection and radiation heat exchange with surrounding," International Journal of Heat and Mass Transfer, vol. 81, pp. 439-448, 2015.

[9] J. Ma, Y. Sun, B. Li, and H. Chen, "Spectral collocation method for radiative-conductive porous fin with temperature dependent properties," Energy Conversion and Management, vol. 111, pp. 279-288, 2016.

[10] J. Ma, Y. Sun, and B. Li, "Simulation of combined conductive, convective and radiative heat transfer in moving irregular porous fins by spectral element method," International Journal of Thermal Sciences, vol. 118, pp. 475-487, 2017.

[11] B. Alshuraiaan and K. Khanafer, "The effect of the position of the heated thin porous fin on the laminar natural convection heat transfer in a differentially heated cavity," International Communications in Heat and Mass Transfer, vol. 78, pp. 190-199, 2016.

[12] A. K. Asl, S. Hossainpour, M. M. Rashidi, M. A. Sheremet, and Z. Yang, "Comprehensive investigation of solid and porous fins influence on natural convection in an inclined rectangular enclosure," International Journal of Heat and Mass Transfer, vol. 133, pp. 729-744, 2019.

[13] R. S. R. Gorla and A. Y. Bakier, "Thermal analysis of natural convection and radiation in porous fins," International Communications in Heat and Mass Transfer, vol. 38, no. 5, pp. 638-645, 2011.

[14] M. Torab and H. Yaghoobi, "Series solution for convectiveradiative porous fin using differential transformation method," Journal of Porous Media, vol. 16, pp. 341-349, 2013.

[15] M. T. Darvishi, R. S. R. Gorla, F. Khani, and B. J. Gireesha, "Thermal analysis of natural convection and radiation in a fully wet porous fin," International Journal of Numerical Methods for Heat \& Fluid Flow, vol. 26, no. 8, pp. 2419-2431, 2016.

[16] M. T. Darvishi, F. Kani, and R. S. R. Gorla, "Natural convection and radiation in a radial porous fin with variable thermal conductivity," International Journal of Applied Mechanics and Engineering, vol. 19, no. 1, pp. 27-37, 2014.

[17] D. A. Nield and A. Bejan, Convection in Porous Media, Springer, Berlin, Germany, 5th edition, 2017.

[18] E. M. Sparrow and R. D. Cess, Radiation Heat Transfer, Hemisphere Publishing Corporation, New York, NY, USA, 1978.

[19] R. M. S. Gama, “An alternative mathematical modelling for coupled conduction/radiation energy transfer phenomenon in a system of gray bodies surrounded by a vacuum," International Journal of Non-linear Mechanics, vol. 30, pp. 433-447, 1995.

[20] P. L. Ndlovu and R. J. Moitsheki, “Thermal analysis of natural convection and radiation heat transfer in moving porous fins," Frontiers in Heat and Mass Transfer, vol. 12, 2019.

[21] M. T. Darvishi, R. S. R. Gorla, and F. Khani, "Natural convection and radiation in porous fins," International Journal of Numerical Methods for Heat \& Fluid Flow, vol. 23, no. 8, pp. 1406-1420, 2013.

[22] V. Vendas Sarmento and M. L. Martins-Costa, "Spectral and finite difference simulations for temperature distribution in a porous fin," in Proceedings of the 17th Brazilian Congress of Thermal Sciences and Engineering, Aguas de Lindóia, São Paulo, Brazil, November 2018.

[23] M. S. Berger, Nonlinearity and Functional Analysis: Lectures on Nonlinear Problems in Mathematical Analysiss, Academic Press, London, UK, 1977.

[24] L. Adams and J. L. Nazareth, Linear and Nonlinear Conjugate Gradient-Related Methods, SIAM, Philadelphia, PA, USA, 1996.

[25] W. H. Press, S. A. Teukolsky, W. T. Vetterling, and B. P. Flannery, Numerical Recipes in Fortran 77: The Art of Scientific Computing, Cambridge University Press, Second edition, 1997. 